\title{
Spaghetti Breaking Dynamics
}

\author{
Yi Zhang*) Xiang Li*) Yuanfan Dai*) Bohua Sun*1)1 \\ 1*(School of Civil Engineering 83 Institute of Mechanics and Technology, \\ Xi'an University of Architecture and Technology, Xi'an 710055, China)
}

\begin{abstract}
Why are pieces of spaghetti generally broken into three to ten segments instead of two as one thinks? How can one obtain the desired number of fracture segments? For these problems, in this paper a strand of spaghetti is considered an elastic rod, and the finite-element software ABAQUS is used to simulate the detailed fracture dynamics of the elastic rod. By changing the size (length and diameter) of the rod, the relevant data on the fracture limit curvature and the number of fractured segments of the elastic rod are obtained. The ABAQUS simulation results confirm the scientific judgment of B. Audoly and S. Neukirch (Fragmentation of rods by cascading cracks: Why spaghetti does not break in half. Phys Rev Lett 95: 095505). Using dimensional analysis to fit the finiteelement data, two relations of the elastic rod fracture dynamics are obtained: (1) the relationship between the fracture limit curvature and the diameter, and (2) the relationship between the number of fracture segments and the diameter-length ratio. Results reveal that when the length is constant, the larger the diameter (the smaller the diameter-length ratio $\mathrm{D} / \mathrm{L}$ ), the smaller the limit curvature; and the larger the diameter-length ratio $\mathrm{D} / \mathrm{L}$, the fewer the number of fractured segments. The relevant formulations can be used to obtain the desired number of broken segments of spaghetti by changing the diameter-to-length ratio.
\end{abstract}

Keywords: curvature, brittle cracking, elastic rod, diameter-to-length ratio, spaghetti

\section{INTRODUCTION}

Numerous objects can be described as elastic rods in ordinary life, such as bones ${ }^{[1]}$ and trees ${ }^{[2-4]}$, and even fiber cells ${ }^{[5]}$ or other models with important physical and physiological functions on various scales. Artificial objects like carbon nanotube composite materials ${ }^{[6]}$, semi-flexible polymer ports ${ }^{[7]}$, and multi-walled carbon nanotubes ${ }^{[8,9]}$ can all be described by the mechanical model of elastic rods as well. Elastic rods are also very common in engineering applications, such as circular cross-section beams and columns in civil engineering ${ }^{[10]}$ and automobile drive shafts ${ }^{[28]}$. During the 2012 London Olympics, Cuban Pole vaulter Lazaro Borges nearly died when the pole broke into three sections at a height of $5 \mathrm{~m}$. The importance of the elastic rod is evident and its ultimate curvature when it is about to break has also become a problem to urgently solve.

Thus, a quasi-static experiment is considered: a piece of dry spaghetti is slowly bent to its limit curvature. In most cases, a strand of spaghetti, which can be regarded as an elastic rod, will not break into two pieces as intuitively thought, but into three to ten pieces. This simple and interesting experiment created Feynman's doubts, and the issue was not resolved at that time ${ }^{[12]}$.

The mechanism of elastic rod fracture and crack propagation has been studied for a long time ${ }^{[11-16]}$. It has been mainly studied from the perspective of statics ${ }^{[17,18]}$. Mott's work ${ }^{[19]}$ in 1945 established the foundation for

Corresponding Author: Bohua Sun.

Email: sunbohua@xauat.edu.cn, http://imt.xauat.edu.cn.

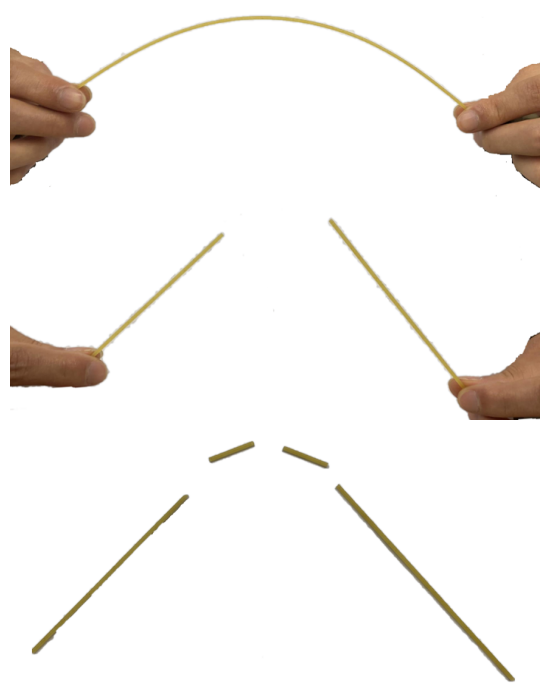

FIG. 1: Spaghetti bending breaking.

the study of the fracture phenomenon of elastic rods. Shockey ${ }^{[20]}$ took the transmission of stress waves as the starting point, and Wittel ${ }^{[21]}$ studied the fragments produced by brittle materials. Mitchell ${ }^{[22]}$ studied the mechanism of curvature affecting the crack direction of materials. Villermaux ${ }^{[23]}$ studied the distribution of energy in brittle materials and the time of the fracture process, and discussed the number of segments and fragment lengths generated. Audoly ${ }^{[12]}$ studied the fracture of a slender rod in the adiabatic state and the transmission of bending waves, while Gladden ${ }^{[13]}$ studied the fracture and energy problems of a slender rod under the condition of rapid heat transfer. 
Fracture phenomena appear in large numbers in life and engineering, and the process of breaking into multiple segments involves many disciplines and technical fields.

In the first part of this paper, simulation of spaghetti curvature and the number of fractured sections in ABAQUS finite-element software are described; in addition, the experiments of Heisser ${ }^{[24]}$ are reproduced and his results verified. In the second part, the data of limit curvature are fitted through dimensional analysis. In the third part, a mathematical model of the fracture dynamics of spaghetti is given, and the coefficient of Audoly is verified as $\frac{\kappa}{\kappa_{0}}=1.428^{[12]}$. In the fourth part, the data of the number of fractures are processed in ABAQUS and the fitting equation given.

\section{SPAGHETTI DYNAMICS SIMULATION}

\section{Finite-element-model analysis}

The ABAQUS finite-element model (FEM) presented in this paper is based on the experiments of Heisser ${ }^{[24]}$ under the conditions of humidity $21 \%-34 \%$ and temperature $21 \%-26 \%$. The length and radius of the spaghetti is $L=240 \mathrm{~mm}$ and $d=0.75 \mathrm{~mm}$, respectively, and its density is $\rho=1.5 \pm 0.1 \mathrm{~g} / \mathrm{cm}^{3}$. The elastic modulus is $E=3.8 \pm 0.3 \mathrm{GPa}$ and Poisson's ratio is $\nu=0.3 \pm 0.1$. The model's data here are $\rho=1.5 \mathrm{~g} / \mathrm{cm}^{3}, E=3.8 \mathrm{GPa}$, and $\nu=0.3$. Villermaux ${ }^{[23]}$ pointed out that the mechanical elastic energy stored in the strained material plays a positive role in promoting fracture and the initial defects in the material proved to be minor. Therefore, this model does not consider the initial defects. Two ends of the spaghetti strand were set to be hinged, and the $10 \mathrm{~mm}$ surface from each end to the reference point were coupled to simulate the case of insertion into the support in the experiments. This model uses units of C3D8R. The schematic of the FEM of the above specimen is shown in Fig. 2.

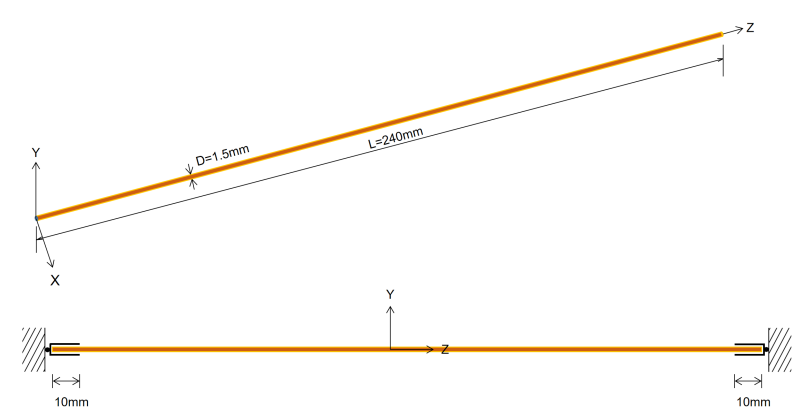

FIG. 2: FEM model.

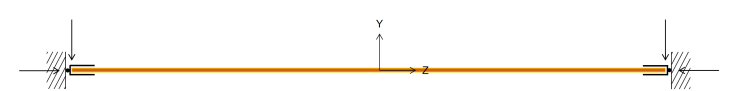

(a)Application of $\mathrm{Y}$ and $\mathrm{Z}$ displacements to both ends of a rod to reach a certain curvature

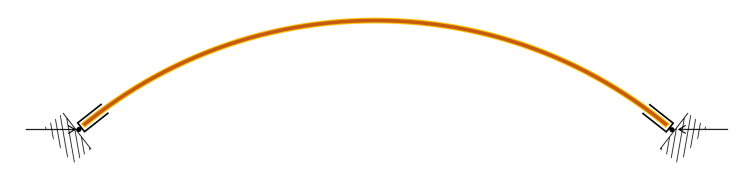

(b)Squeezing of two ends of a rod at a certain speed to the limit curvature

FIG. 3: Loading process.

Heisser's experiments used the quench speed of the two ends as the variable in the experiments. However, directly squeezing both ends is likely to cause the stability and buckling problem ${ }^{[29]}$, discussion of which is not within the scope of this paper; Heisser's experiments also exclude the relevant results.

In the present work, $\mathrm{Y}$ and $\mathrm{Z}$ displacements were first applied to both ends of the rod to reach a certain curvature, and then two ends of the rod squeezed at a certain speed to the limit curvature, as shown in Fig. 3. See the attachment for a description of the complete dynamic fracture process.

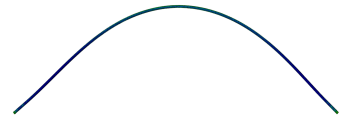

(a)Bending to limit curvature $\mathrm{t}=0$

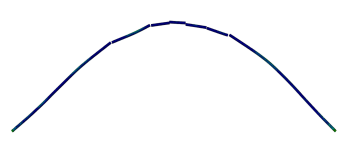

(c) $\mathrm{t}=0.3 \mathrm{~ms}$

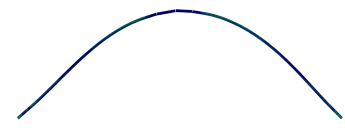

(b) $\mathrm{t}=0.1 \mathrm{~ms}$

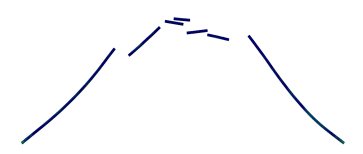

(d) $\mathrm{t}=1.9 \mathrm{~ms}$
FIG. 4: Dynamic fracture process of spaghetti.

\section{Simulation of limit curvature}

Discussion of the limit curvature was facilitated by changing the size of the spaghetti with length $L=$ $240 \mathrm{~mm}$ and by increasing the diameter from 1 to $3 \mathrm{~mm}$ in increments of $0.1 \mathrm{~mm}$ to achieve different diameters in order to study the relationship between the diameter-tolength ratio and limit curvature. The relationship obtained by the FEM is shown in Fig. 5. It can be seen from the figure that the limit curvature exhibits an obvious trend with the change of the diameter-length ratio. 
These data are discussed and analyzed aided by dimensional analysis in Section 2.

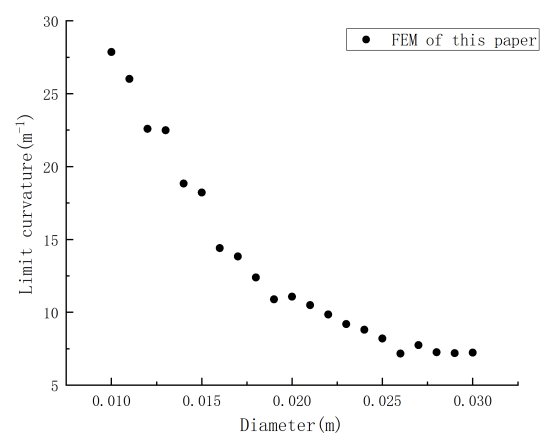

FIG. 5: Relationship between limit curvature and diameter.

\section{Simulation of dynamic process after fracture}

For the dynamic process after spaghetti fracture, in this paper the fracture of a rod when the length is $L=240 \mathrm{~mm}$, diameter is $D=1.5 \mathrm{~mm}$, and quench speed is $v=300 \mathrm{~mm} / \mathrm{s}$ is taken as the object of study. From Figs. 6 and 7 , it can be seen that when $t=0$, point a is broken, $\kappa_{a}=7.968 \mathrm{~m}^{-1}$, when $t=0.0017 \mathrm{~s}$, the curvature of point $\mathrm{b}$ is $\kappa_{b}=11.571 \mathrm{~m}^{-1}$. In Section 3 , the relationship between the curvature of these two points and the dynamic process after fracture is discussed in detail.

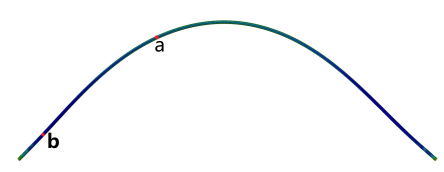

(a)Spaghetti has been bent to its extreme curvature

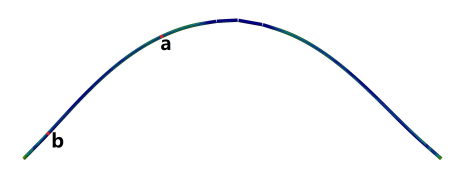

(b)Point of maximum curvature has begun to fracture; at this time point a has not yet fractured

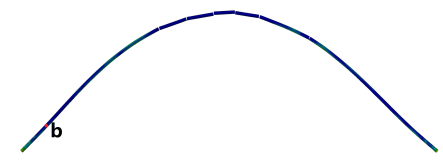

(c)Point a breaks at $t=0$

FIG. 6: Using FEM to study dynamic process of spaghetti fracture.

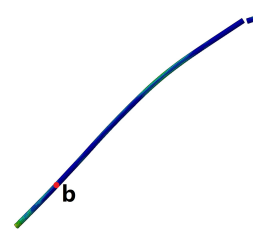

(a) $t=0$

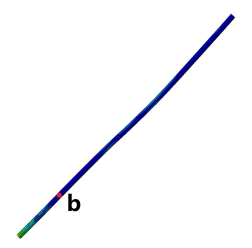

(c) $t=0.0008 \mathrm{~s}$

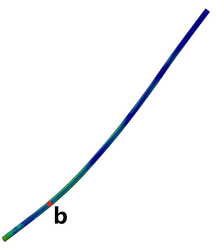

(e) $\mathrm{t}=0.0016 \mathrm{~s}$

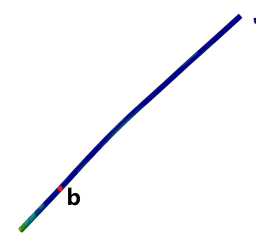

(b) $t=0.0005 \mathrm{~s}$

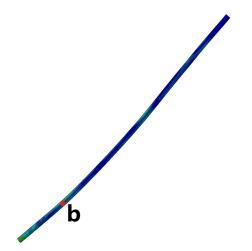

(d) $t=0.0012 s$

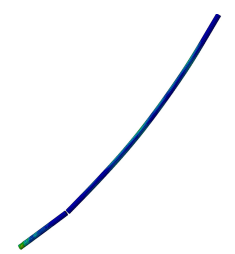

(f)Point b breaks at $t=0.0017 \mathrm{~s}$
FIG. 7: Using FEM to study dynamic process of spaghetti fracture.

\section{Simulation of number of fractured sections}

For the simulation of the number of fractured segments of spaghetti, the model is taken at the point when the lengths of the spaghetti are $L=200,220$, and $240 \mathrm{~mm}$ and the diameter $D$ ranges from 1 to $3 \mathrm{~mm}$. The fractured segment number is used to obtain the relationship between the fractured segments. When the ratio of diameter to length is the same, the number of fractured segments would be averaged, as shown in Fig. 8. It can be seen from the figure that the number of fractured segments gradually converges to a constant as the diameterto-length ratio increases. The data are discussed and fitted in Section 4.

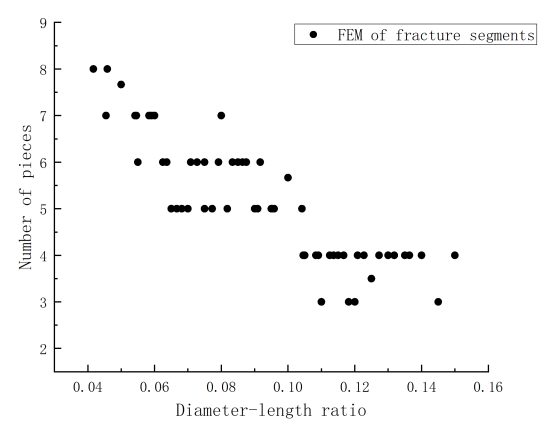

FIG. 8: Relationship between fractured segment number and diameter-to-length ratio. 


\section{Simulation verification of experiment}

By changing the relative quench speed at loading both ends as follows, 1, 2, 3, 5, 10, 20, 30, 50, 100, 200, 300, and $500 \mathrm{~mm} / \mathrm{s}$, different limit curvatures and fracture segment numbers are obtained. After collating the data and comparing them with those from Heisser's experiment, the result is shown in Fig. 9.

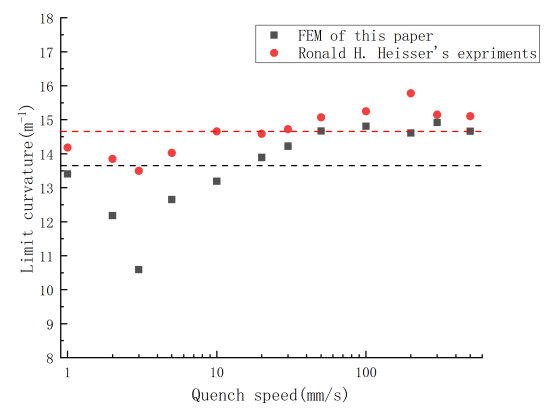

FIG. 9: Red dotted line represents average limit curvature of Heisser's experimental results and black dotted line represents average limit curvature of FEM used in the present paper.

The difference between average limit curvature obtained by Heisser and FE simulation being $6.9 \%$. The difference may be caused by the imperfect support conditions in the experiments and the non-uniformity of the spaghetti. The trend of the number of fractured segments is the same as that in the experiments, but the number of fractured segments obtained by the experiment tends to three while that obtained by the FEM is between three and four. This may be caused by the boundary conditions imposed by the FEM being ideal and completely symmetrical, so the number of fractured segments tend$\mathrm{s}$ to be even, so that there are more results that tend to produce four segments ${ }^{[2]}$. Different quench speeds have little effect on the limit curvature, which is consistent with Heisser's experimental results and conclusions, which verifies the reliability of the FEM.

\section{DIMENSIONAL ANALYSIS AND CURVATURE FITTING}

To discuss the limit curvature and fit the data in Fig. 5 , through the theory of dimensional analysis ${ }^{[26,27]}$, six parameters of the elastic rod can be listed, all six of which have three dimensions ( $\mathrm{L}, \mathrm{M}$, and $\mathrm{T}$ ), as shown in Table 1.

As a result, this problem produces $6-3=3$ dimensionless quantities $\Pi$, as follows:

$\Pi_{1}=L D^{a} \rho^{b} \sigma_{c}^{c}, \quad \Pi_{2}=E D^{a_{1}} \rho^{b_{1}} \sigma_{c}^{c_{1}}, \quad \Pi_{3}=\kappa D^{a_{2}} \rho^{b_{2}} \sigma_{c}^{c_{2}}$.
Table 1 Basic dimensions ( mass $(\mathrm{M})$, length $(\mathrm{L})$, and time $(\mathrm{T})$ )

\begin{tabular}{l|l|l}
\hline \hline Variable & Symbol & Dimension \\
\hline Diameter & $\mathrm{D}$ & $\mathrm{L}$ \\
\hline Length & $\mathrm{L}$ & $\mathrm{L}$ \\
\hline Density & $\rho$ & $M L^{-3}$ \\
\hline Elastic modulus & $\mathrm{E}$ & $M L^{-1} T^{-2}$ \\
\hline Critical stress & $\sigma_{c}$ & $M L^{-1} T^{-2}$ \\
\hline Limit curvature & $\kappa$ & $L^{-1}$ \\
\hline \hline
\end{tabular}

a,b,c, $a_{1}, b_{1}, c_{1}$, and $a_{2}, b_{2}, c_{2}$ can be obtained by the dimensionless condition $\operatorname{dim}\left(\Pi_{1}\right)=\operatorname{dim}\left(\Pi_{2}\right)=$ $\operatorname{dim}\left(\Pi_{3}\right)=D^{0} \rho^{0} \sigma_{c}^{0}$ :

$$
\begin{aligned}
\operatorname{dim}\left(\Pi_{1}\right) & =L L^{a} M^{b} L^{-3 b} M^{c} L^{-c} T^{-2 c} \\
& =L^{1+a-3 b-c} M^{b+c} T^{-2 c}
\end{aligned}
$$

and with $1+\mathrm{a}-3 \mathrm{~b}-\mathrm{c}=0, \mathrm{~b}+\mathrm{c}=0$, and $-2 \mathrm{c}=0$, one can obtain $\mathrm{a}=-1, \mathrm{~b}=0$, and $\mathrm{c}=0$. Bringing in the determined $\mathrm{a}$, $\mathrm{b}$, and $\mathrm{c}$, the first dimensionless quantity can be obtained as follows:

$$
\begin{aligned}
& \Pi_{1}=\frac{L}{D}, \\
& \Pi_{1, \text { modified }}=\frac{D}{L},
\end{aligned}
$$

where $\Pi_{1}$ has been rewritten into $\Pi_{1, \text { modified }}$, which is the diameter-to-length ratio.

In the same way, one can obtain $a_{1}=0, b_{1}=0$, and $c_{1}=-1$, and the second dimensionless quantity is

$$
\begin{aligned}
& \Pi_{2}=\frac{E}{\sigma_{c}}, \\
& \Pi_{2, \text { modified }}=\frac{\sigma_{c}}{E}=\epsilon_{c},
\end{aligned}
$$

where $\Pi_{2}$ has also been rewritten into $\Pi_{2, \text { modified }}$, which means the ultimate strain, i.e., the ratio of ultimate stress to the elastic modulus.

Similarly, one obtains $a_{2}=1, b_{2}=0$, and $c_{2}=0$, so the third dimensionless quantity is

$$
\Pi_{3}=\kappa D
$$

According to dimensional analysis theory, one can obtain $\Pi_{3}=f\left(\Pi_{1, \text { modified }}, \Pi_{2, \text { modified }}\right)$, which could be written as

$$
\kappa D=f\left(\frac{D}{L}, \epsilon_{c}\right) .
$$

Assuming that the ultimate strain of spaghetti is fixed, then $\epsilon_{c}$ is a constant and Eq. (6) can be rewritten as 


$$
\kappa=\frac{1}{D} f\left(\frac{D}{L}\right)
$$

According to Barenblat's suggestion ${ }^{[31]}, f\left(\frac{D}{L}\right)$ can be changed to an expansion similar to a power series:

$$
f\left(\frac{D}{L}\right)=C\left(\frac{D}{L}\right)^{\alpha}
$$

where $\mathrm{C}$ and $\alpha$ are constants. Inserting Eq. (8) into Eq. (7)

$$
\kappa=C \frac{D^{\alpha-1}}{L^{\alpha}} .
$$

Since $L=240 \mathrm{~mm}$, the constants $\mathrm{C}$ and $\alpha$ can be determined by data fitting. Through FE data fitting, the relationship between the limit curvature and diameter of spaghetti at a humidity of $21 \%-34 \%$, temperature of $21 \%-26 \%$, and quench speed of $300 \mathrm{~mm} / \mathrm{s}$ can be obtained; in addition, $C=0.091$ and $\alpha=-0.366$, as shown in Fig. 10:

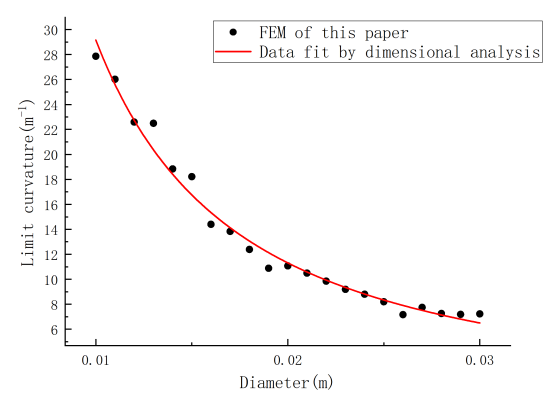

FIG. 10: Obtaining fitting curve of limit curvature through dimensional analysis.

$$
\kappa=0.676 D^{-1.366}
$$

The relationship between the radius and limit curvature when the length of the spaghetti is $L=240 \mathrm{~mm}$ is thus obtained.

\section{DYNAMIC MATHEMATICAL MODEL AFTER FRACTURE}

Audoly believes that the multi-segment fracture of spaghetti is due to the cascading effect caused by the transmission of bending waves after the first fracture. Taking Fig. 6(c) as an example, the fractured spaghetti is divided into three parts designated a, b, and c. Sections a and c can be regarded as the cantilever cases, and section $b$ denotes the spaghetti segments with two free ends. Only the cantilever situation is discussed here, as shown in Figs. 10(a) and 10(c). Section a is taken as an example.

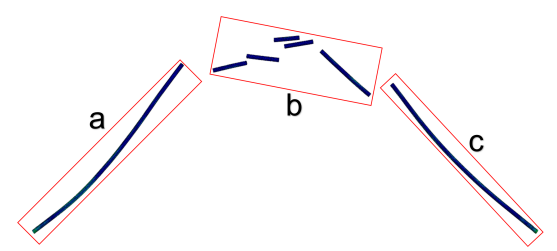

FIG. 11: Sections a and c are cantilever beams; section a is taken as an example.

The Euler-Bernoulli beam equation ${ }^{[30]}$ can be applied to the spaghetti after breaking:

$$
\frac{\partial^{4} y}{\partial s^{4}}+\frac{\rho A}{E I} \frac{\partial^{2} y}{\partial t^{2}}=0,
$$

where $y$ is the deflection, $s$ the arc length, $t$ the time, $I$ the moment of inertia of the section, and $A$ the crosssectional area of the rod. Taking the double derivative of the arc length s on both sides of Eq. (11), one obtains $E I \frac{\partial^{4}}{\partial s^{4}}\left(\frac{\partial^{2} y}{\partial s^{2}}\right)+\rho A \frac{\partial y}{\partial t^{2}}\left(\frac{\partial^{2} y}{\partial s^{2}}\right)=0$. Using the definition of curvature, $\kappa=\frac{\partial^{2}}{\partial s^{2}} y(s, t)$, Eq. (11) can be rewritten as

$$
E I \frac{\partial^{4} \kappa}{\partial s^{4}}+\rho A \frac{\partial^{2} \kappa}{\partial t^{2}}=0
$$

The self-similarity solution of Eq. (13) is

$$
\begin{aligned}
\kappa(s, t) & =C_{1} \frac{s^{2}}{t} \text { hypergeom }\left(\left[\frac{1}{2}, 1\right],\left[\frac{3}{4}, \frac{5}{4}, \frac{3}{2}\right],-\frac{\rho A s^{4}}{64 E I t^{2}}\right) \\
& +C_{2} \text { FresnelS }\left[\frac{1}{\sqrt{2 \pi}}\left(\frac{\rho A}{E I}\right)^{\frac{1}{4}} \frac{s}{\sqrt{t}}\right] \\
& +C_{3} \text { FresnelC }\left[\frac{1}{\sqrt{2 \pi}}\left(\frac{\rho A}{E I}\right)^{\frac{1}{4}} \frac{s}{\sqrt{t}}\right]+C_{4},
\end{aligned}
$$

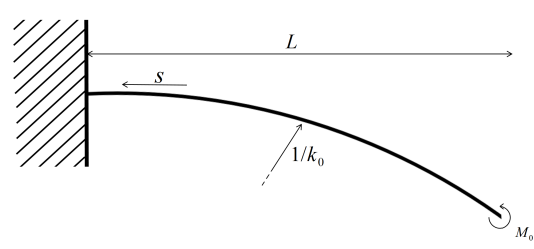

FIG. 12: Spaghetti model after first spaghetti break.

where $C_{1}, C_{2}, C_{3}$, and $C_{4}$ are undetermined coefficients. The place $s=0$ is the free end, $s=L$ is the fixed end, and the boundary conditions are brought in as $\kappa(0, t)=0, \kappa(s, 0)=\kappa_{0}, \frac{\partial \kappa}{\partial s}(0, t)=0$, and $\frac{\partial^{2} \kappa}{\partial s^{2}}(0, t)=0\left(\kappa_{0}\right.$ is the initial curve), after which one obtains $C_{1}=C_{3}=C_{4}=0, C_{2}=2 \kappa_{0}$. 
Substituting $C_{1}=C_{3}=C_{4}=0, C_{2}=2 \kappa_{0}$ into Eq. (13), and then regarding $\xi=\left(\frac{\rho A}{E I}\right)^{\frac{1}{4}} \frac{s}{\sqrt{t}}$ as a selfsimilarity variable, Eq. (14) is rewritten as

$$
\boldsymbol{\kappa}(s, t)=2 \kappa_{0} \text { FresnelS }\left(\frac{\xi}{\sqrt{2 \pi}}\right) .
$$

Eq. (14) is the expression of the curvature of spaghetti after breaking.

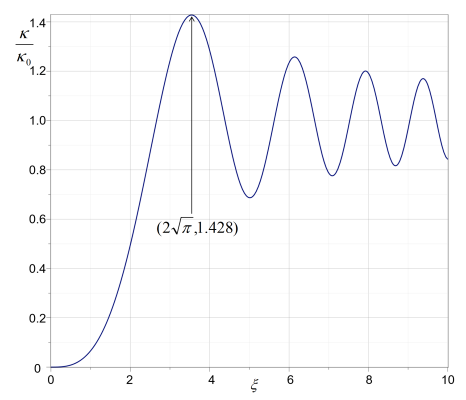

FIG. 13: Obtaining function image $\frac{\kappa}{\kappa_{0}}=2$ FresnelS $\left(\frac{\xi}{\sqrt{2 \pi}}\right)$, which is easy to know when $\xi=2 \sqrt{\pi}, \frac{\kappa}{\kappa_{0}}$ could reach the maximus of 1.428 .

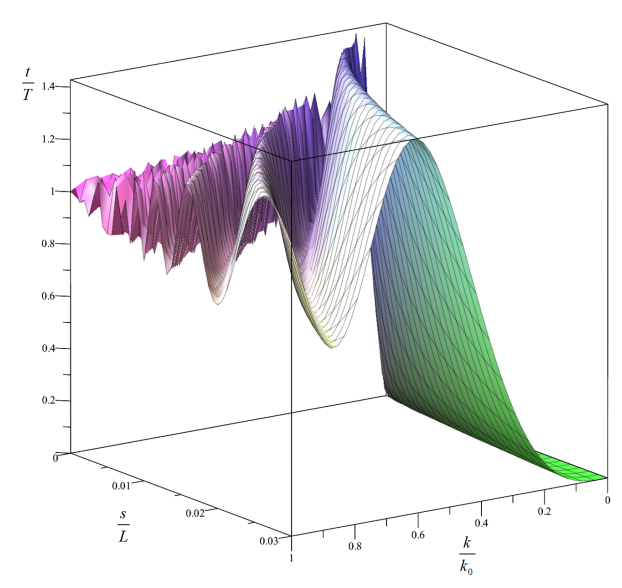

FIG. 14: Numerical solution of Eq. (15) under the boundary condition that one end is fixed and the other is free. It can be seen that the curvature of the free end $\kappa$ tends to zero in a short time.

Figure 13 shows that, when $\xi=2 \sqrt{\pi}$, FresnelS $\left(\frac{\xi}{\sqrt{2 \pi}}\right)$ reached the maximum value; at this time, the curvature $\kappa$ is 1.428 times the initial curvature $\kappa_{0}$. Audoly believes that the coefficient 1.428 is universal ${ }^{[12]}$, which is double the maximum value of the Fresnel sine integral. The coefficient of $\frac{\kappa}{\kappa_{0}}=1.428$ obtained by the mathematical model through simulation was also verified in this article, and the corresponding finite element is shown in Figs. 6 and 7. For a piece of spaghetti that has been bent to the limit curvature, the end that was broken could be regarded as the free end that is released, and the other end is $10 \mathrm{~mm}$ long for plug-in articulation in this paper. The inserted part can be regarded as a fixed end relative to the other parts of the spaghetti, so it meets the boundary and initial conditions; this process does not involving any material properties. The curvature $\kappa_{a}$ of the breaking point a at $t=0$ relaxed in a short period of time. This sudden relaxation produces an explosive stress wave, strong enough to break the remaining part at this time, $\kappa_{a}=7.968 \mathrm{~m}^{-1}$. When $t=0.0017 \mathrm{~s}, \kappa_{b}=11.571 \mathrm{~m}^{-1}$ at point $\mathrm{b}$, and point $\mathrm{b}$ breaks when $\mathrm{t}=0.0018 \mathrm{~s}$. In this process, $\kappa_{b}$ is 1.452 times $\kappa_{a}$. The difference between 1.452 and 1.428 is $1.7 \%$. This also supports Audoly's $\frac{\kappa}{\kappa_{0}}=1.428$ coefficient.

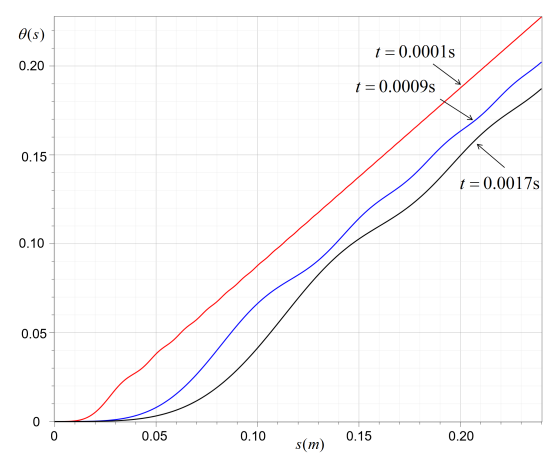

FIG. 15: After the first break (released as a free end) at $\mathrm{t}=0.0001,0.0009$, and $0.0017 \mathrm{~s}$, the relationship between $\theta$ and $s$ is obtained.

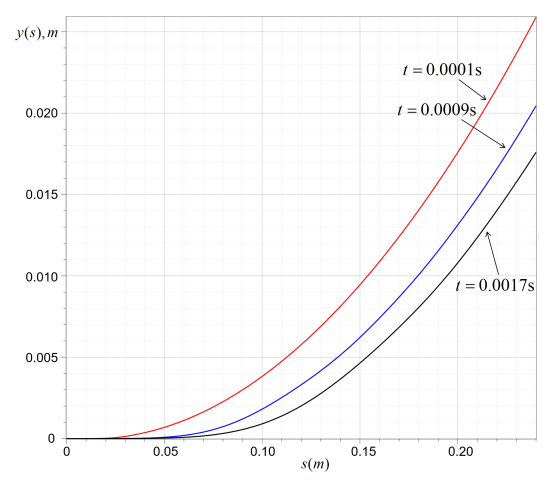

FIG. 16: After the first break (released as a free end) at $\mathrm{t}=0.0001,0.0009$, and $0.0017 \mathrm{~s}$, the relationship between $y$ and $s$ is obtained.

\section{DIMENSIONAL ANALYSIS AND FITTING OF FRACTURED SEGMENT NUMBER}

Audoly's work ${ }^{[12]}$ may be able to give the specific fracture time and location without using any specific fracture criteria, but to obtain a simple and universal law that 
could be convenient in engineering applications, the following lengths of the spaghetti model are taken, $\mathrm{L}=200$, 220 , and $240 \mathrm{~mm}$, when the diameter $D$ is in the range of 1 to $3 \mathrm{~mm}$. The relationship between the fractured segment number and the diameter-to-length ratio are then discussed. When the diameter-to-length ratios are the same, the number of fractured segments is averaged, as shown in Fig. 17.

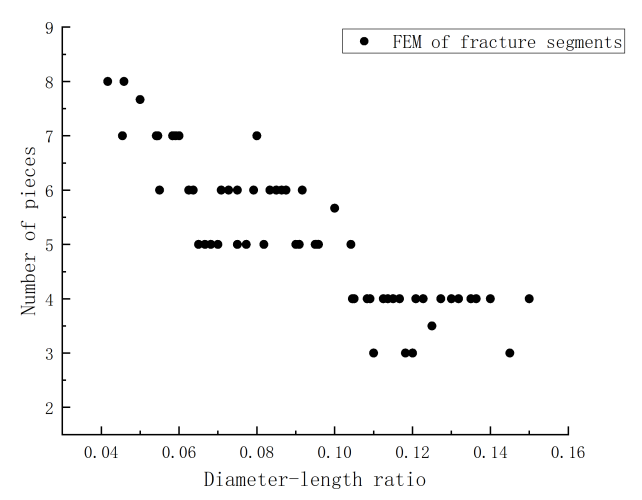

FIG. 17: Relationship between number of fracture segments and diameter-to-length ratio.

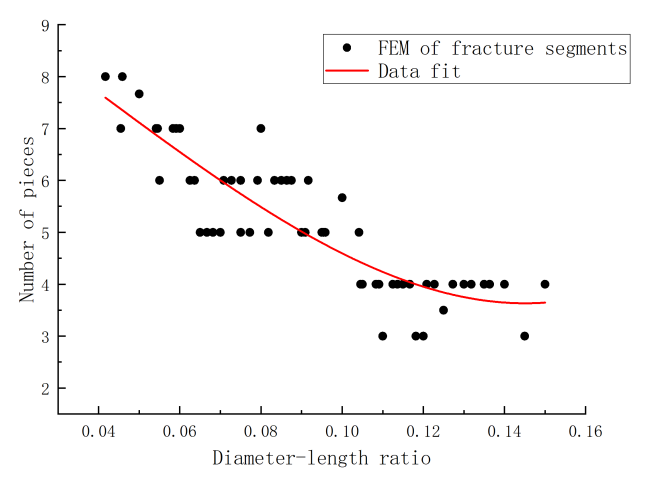

FIG. 18: Fitting-curve diagram of fracture segment number and diameter-to-length ratio.

It can be seen that the number of fracture segments gradually converges to a value with increasing diameterto-length ratio. To study the relationship between them, polynomial fitting is performed on the FE data, and the formulation is

$$
N=\alpha\left(\frac{D}{L}\right)+\beta\left(\frac{D}{L}\right)^{2}+\gamma\left(\frac{D}{L}\right)^{3}+\delta
$$

Fitting to obtain $\alpha=1760.41, \beta=-214.25, \gamma=$ -49.05 , and $\delta=9.88$ gives

$$
N \approx 1760.41\left(\frac{D}{L}\right)-214.25\left(\frac{D}{L}\right)^{2}-49.05\left(\frac{D}{L}\right)^{3}+9.88
$$

Drawing the fitting curve together with the FE data, the plot shown in Fig. 18 is obtained.

It can be seen that the number of fractured segments converges to 3.6 with diameter-to-length ratio. This is an easy rule to apply.

\section{CONCLUSIONS}

In terms of fracture limit curvature, the relationship between fracture limit curvature and diameter is obtained through dimensional analysis as exemplified by Eq. (9). After fitting the FE simulation data, it was found that when the spaghetti length $\mathrm{L}$ is constant, the limit curvature is inversely proportional to the diameter of the elastic rod, and the relationship between them is expressed by Eq. (10).

In terms of the dynamic mathematical model after fracture, the Euler-Bernoulli beam equation and FE method are used in this paper to confirm the conclusion of Audoly that $\frac{\kappa}{\kappa_{0}}=1.428$. This conclusion can be used to theoretically predict the time and location of the next cantilever break based on the initial conditions after the first break.

In terms of the number of fractures, the occurrence of fracture segment numbers when the spaghetti lengths are $\mathrm{L}=200,220$, and $240 \mathrm{~mm}$ and the diameters $D$ are in the range of 1 to $3 \mathrm{~mm}$ was simulated. The FE simulation results show that the fracture segment number of segments gradually decreases from eight with increasing diameterto-length ratio, and converges to 3.6. The relationship between them is expressed by Eq. (16). The decreasing trend of their fracture segments is in line with the actual law.

In this paper, the length of the spaghetti selected and value range of the diameter does not exert much influence. If more simulations under different sizes and more data can be obtained, it is our belief that the limitcurvature-to-diameter-length ratio can be obtained. The relationship between the number of fractured segments and diameter-to-length ratio is more accurate.

[1] Peterlik H, Roschger P, Klaushofer K, Fratzl P (2006) From brittle to ductile fracture of bone. Nat Mater 5:5255.

[2] Gardiner B, Berry P, Moulia B (2016) Review: Wind impacts on plant growth,mechanics and damage. Plant Sci 245:94-118.

[3] Virot E, Ponomarenko A, Dehandschoewercker É, Quéré D , Clanet C (2016) Critical wind speed at which trees break. Phys Rev E 93:023001.

[4] Albrecht A, et al. (2016) Comment on critical wind speed at which trees break. Phys Rev E 94:067001. 
[5] Odde DJ, Ma L, Briggs AH, DeMarco A, Kirschner MW (1999) Microtubule bending and breaking in living fibroblast cells. J Cell Sci 112:3283C3288.

[6] Baughman RH, Zakhidov AA, de Heer WA (2002) Carbon nanotubes-The route toward applications. Science 297:787C792.

[7] Kantsler V, Goldstein RE (2012) Fluctuations, dynamics, and the stretch-coil transition of single actin filaments in extensional flows. Phys Rev Lett 108:038103.

[8] Yu MF, et al. (2000) Strength and breaking mechanism of multiwalled carbon nanotubes under tensile load. Science 287:637C640.

[9] Mokashi VV, Qian D, Liu Y (2007) A study on the tensile response and fracture in carbon nanotube-based composites using molecular mechanics. Compos Sci Technol 67:530-540.

[10] Hetnyi M (1946) Beams on Elastic Foundation: Theory with Applications in the Fields of Civil and Mechanical Engineering (Univ Michigan Press, Ann Arbor, MI).

[11] Bouchaud E (1997) Scaling properties of cracks. J Phys Condens Matter 9:4319-4344.

[12] Audoly B, Neukirch S (2005) Fragmentation of rods by cascading cracks: Why spaghetti does not break in half. Phys Rev Lett 95:095505.

[13] Gladden JR, Handzy NZ, Belmonte A, Villermaux E (2005) Dynamic buckling and fragmentation in brittle rods. Phys Rev Lett 94:035503.

[14] Herrmann HJ, Hansen A, Roux S (1989) Fracture of disordered, elastic lattices in two dimensions. Phys Rev B 39:637-648.

[15] Peerlings RHJ, de Borst R, Brekelmans WAM, Geers MGD (1998) Gradient-enhanced damage modelling of concrete fracture. Mech Cohes-Frict Mat 3:323-342.

[16] Gerstle W, Sau N, Silling S (2007) Peridynamic modeling of concrete structures. Nucl Eng Des 237:1250-1258.

[17] D. E. Grady and M. E. Kipp, J. Appl. Phys. 58, 1210(1985).

[18] R. Englman, J. Phys. Condens. Matter 3, 1019 (1991).

[19] Mott N (1947) Fragmentation of shell cases. Proc R Soc
Lond A Math Phys Sci 189:300-308.

[20] D. A. Shockey, D. R. Curran, L. Seaman, J. T. Rosenberg, and C. F. Peterson, Int. J. Rock Mech. Min. Sci.Geomech. Abstr. 11, 303 (1974).

[21] Wittel F, Kun F, Herrmann HJ, Kröplin BH (2004) Fragmentation of shells. Phys Rev Lett 93:035504.

[22] Mitchell NP, Koning V, Vitelli V, Irvine WTM (2017) Fracture in sheets draped on curved surfaces. Nat Mater 16:89C93.

[23] Villermaux E (2017) Self-activated fragmentation. Int J Fract 206:171C193.

[24] Heisser RH, Patil VP, Stoop N, Villermaux E, Dunkel J. Controlling fracture cascades through twisting and quenching. Proc Natl Acad Sci U S A. 2018 Aug 28;115(35):8665-8670. doi: 10.1073/pnas.1802831115. Epub 2018 Aug 13. PMID: 30104353; PMCID: PMC6126751.

[25] B. D. Coleman, E. H. Dill, M. Lembo, Z. Lu, and I. Tobias,Arch. Ration. Mech. Anal. 121, 339 (1993).

[26] B. H. Sun, Dimensional Analysis and Lie Group (China High Education Press,Beijing, 2016).

[27] B. H. Sun, Scaling law for the propagation speed of domino toppling. AIP Advances, 2020,10(9),095124

[28] Wei Li Sun, Sen Zhang, Wei Wei Ge, et al. Structure Analysis of Inner Cylinder Components of Drum Washing Machine Based on Ansys/Workbench. 2013, 2601:137-140.

[29] E. Villermaux, K. Keremidis, N. Vandenberghe, M.J. Abdolhosseini Qomi, and F.-J. Ulm (2021) Mode Coarsening or Fracture: Energy Transfer Mechanisms in Dynamic Buckling of Rods. Phys. Rev. Lett. 126, 045501

[30] Bauchau, O. A., and J. I. Craig. "Euler-Bernoulli beam theory." Structural analysis. Springer, Dordrecht, 2009. 173-221.

[31] G. I. Barenblatt, Similarity, Self-Similarity, and Intermediate Asymptotics (Consultants Bureau, New York, 1979). 